\title{
Efeitos de procedimentos para maximizar o bem-estar e a competência parental em mulheres vitimizadas
}

\author{
Effects of procedures to maximize well-being and \\ parental competence in victimized women
}

\author{
Paolla Magioni SANTINI ${ }^{1}$ \\ Lúcia Cavalcanti de Albuquerque WILLIAMS ${ }^{1}$
}

\begin{abstract}
Resumo
O Projeto Parceria visa ensinar habilidades parentais a mulheres com histórico de violência pelo parceiro íntimo, incluindo dois módulos com manuais correspondentes: (I) um específico para analisar os efeitos da violência e seu enfrentamento, e (II) outro para o ensino de manejo positivo de comportamento dos filhos. O presente estudo avaliou os efeitos de três procedimentos de aplicação dos referidos módulos: (a) Módulo I seguido do Módulo II; (b) Módulo II seguido do Módulo I; e (c) Módulo I e II aplicados simultaneamente, em um delineamento intrassujeitos. Nove mães com histórico de violência pelo parceiro íntimo foram distribuídas em três grupos, cada um com um tipo de procedimento, e participaram de 20 sessões individuais. Foram observados efeitos favoráveis da intervenção nos três grupos avaliados, especialmente no que recebeu o procedimento simultâneo de módulos (c).
\end{abstract}

Palavras-chave: Estilo parental; Intervenção psicológica; Violência contra a mulher; Violência doméstica.

\begin{abstract}
The "Projeto Parceria" (The Partnership Project) aims at teaching parenting skills to women with a history of intimate partner violence, including two distinctive units: (I) specific unit to analyze violence effects and its prevention and (II) unit to teach strategies for the effective management of their children's behavior. This paper evaluated the effects of three different procedures used in the application of these units: (a) Unit I followed by Unit II; (b) Unit II followed by Unit I; and (c) Units I and II simultaneously applied using a single-subject design. Nine mothers with a history of intimate partner violence were distributed into three groups, each with one type of procedure, and they participated in 20 individual sessions. Favorable intervention effects were observed in the three groups, especially in the simultaneous units' procedure group (c).
\end{abstract}

Keywords: Parenting style; Psychological intervention; Violence against women; Domestic violence.

\footnotetext{
$\checkmark \nabla \nabla$

1 Universidade Federal de São Carlos, Centro de Educação e Ciências Humanas, Programa de Pós-Graduação em Psicologia. Rod. Washington Luís, Km 235, SP-310, 13565-905, São Carlos, SP, Brasil. Correspondência para/Correspondence to: P.M. SANTINI. E-mail: <paollams@gmail.com>.

Artigo elaborado a partir da dissertação de P.M. SANTINI, intitulada "Eficácia de procedimentos para maximizar bem-estar e competência parental em mulheres vitimizadas". Universidade Federal de São Carlos, 2011.

Apoio: Fundação de Amparo à Pesquisa do Estado de São Paulo (Processo no 2008/07997-2).
} 
Estudos apontam que estilos parentais inadequados são desenvolvidos, em parte, pelo aprendizado do modelo comportamental dos pais de origem. Dessa forma, aqueles que tiveram um histórico de agressão na infância tendem a reproduzir esse modelo com seus filhos (Belsky, Conger, \& Capaldi, 2009; Serbin \& Karp, 2003; Weber, Selig, Bernardi, \& Salvador, 2006). Considerando a relevância da interação entre pais e filhos para o desenvolvimento da criança, é fundamental analisar o modelo parental e a possibilidade da ocorrência de violência intrafamiliar ao se planejar uma intervenção.

Um exemplo da transmissão de práticas inadequadas entre gerações pode ser observado na interface entre a presença de um histórico de violência pelos pais na infância, sofrer violência pelo parceiro íntimo na vida adulta e subsequentes casos de maus-tratos de mães vítimas de violência pelo parceiro íntimo contra seus filhos (Appel \& Holden, 1998; Casanueva, Martin, \& Runyan, 2009; Levendosky, Leahy, Bogat, Davidson, \& Von Eye, 2006; O'Leary, Slep, \& O'Leary, 2000). Esse contexto salienta a necessidade de avaliações globais por parte das instituições as quais atendem mulheres com esse perfil/histórico. Não cabe oferecer cuidados exclusivamente para a mulher, é preciso voltar o olhar também para a condição de seus filhos, investigando a possibilidade de maus-tratos e auxiliando a mãe a educá-los de maneira não violenta, promovendo, por exemplo, intervenções, as quais envolvam a discussão da violência contra a mulher e sua relação com o maltrato infantil.

Dessa maneira, a intervenção psicoterapêutica com mães vítimas de violência pelo parceiro íntimo, visando orientá-las no que diz respeito à educação adequada de seus filhos, pode ser uma das estratégias para a prevenção da violência intrafamiliar e suas consequências nocivas. No entanto, estudos de revisão realizados por Sullivan (2006) e Williams et al. (2010) identificaram apenas um estudo, o de Jouriles et al. (2009), com esse foco.

Diante da escassez de pesquisas de intervenção para prevenir problemas de comportamento em filhos de mulheres vítimas de violência pelo parceiro ciamento do Conselho Nacional de Desenvolvimento Científico e Tecnológico. Posteriormente, o Projeto Parceria foi incorporado ao programa de estágio supervisionado desenvolvido na Universidade (em ambulatório de saúde) e em Casa Abrigo (Williams et al., 2010).

A intervenção possui um pressuposto de acolhimento às mães, que são consideradas como parceiras do profissional e não subalternas. O Projeto Parceria utiliza a abordagem Cognitivo-comportamental e inclui dois módulos: (I) um específico para analisar os efeitos da violência e seu enfrentamento e (II) outro para o ensino de manejo positivo de comportamento dos filhos. Pressupunha-se que a aplicação inicial do Módulo I seria fundamental para lidar com os aspectos emocionais associados ao histórico de violência e outras experiências traumáticas, de forma a gerar autoconhecimento e, assim, contribuir para a aplicação posterior do Módulo II (Williams et al., 2010). Embora a aplicação do Projeto Parceria, considerando a sequência Módulo I seguido de Módulo II, tenha apresentado resultados positivos (Pereira, D'Affonseca, \& Williams, 2013; Williams et al., 2010; Williams, Santini, \& D'Affonseca, 2012, 2014), questiona-se quais seriam os efeitos da aplicação de outras possibilidades de sequenciamento dos módulos.

O objetivo deste estudo consistiu em avaliar os efeitos de três procedimentos diferentes de intervenção a mulheres com histórico de violência pelo parceiro íntimo em termos das variáveis bem-estar (Módulo I) e competência parental (Módulo II), sendo eles: (a) Módulo I seguido do Módulo II; (b) Módulo II seguido do Módulo l; e (c) Módulo I e II aplicados simultaneamente. Pretendeu-se verificar qual tipo de intervenção apresentaria resultados mais favoráveis e, assim, propor uma metodologia que pudesse ser disseminada em outras organizações de apoio a mulheres com esse perfil.

\section{Método}

\section{Participantes}

A partir de encaminhamentos ao ambulatório de saúde da Universidade e ao programa de 
estágio supervisionado, foram selecionadas nove mães que apresentaram queixa relacionada ao histórico de violência pelo parceiro. Para participar da pesquisa, as mães deveriam ter pelo menos um filho com até 12 anos de idade e não apresentar psicopatologia severa (e.g., esquizofrenia).

\section{Instrumentos}

a) Entrevista Inicial com Mães Vítimas de Violência Doméstica (Williams, 2010): a qual contempla dados de identificação; infância; histórico da violência sofrida pelo parceiro; autoimagem; relacionamento com parceiro e com os filhos; e condições de saúde;

b) Inventário de Estilos Parentais (IEP) (Gomide, 2006): composto por 42 questões que avaliam oito práticas educativas (monitoria positiva, comportamento moral, punição inconsistente, negligência, disciplina relaxada, monitoria negativa e abuso físico);

c) The Child Abuse Potencial Inventory (CAP, Inventário de Potencial de Abuso Infantil) (Milner, 1994): o CAP é um inventário composto por 160 afirmações para verificar o potencial de abuso físico dos pais em relação aos filhos. No Brasil foram realizados estudos psicométricos de adaptação de tal instrumento por Patrian, Rios e Williams, 2013 e Rios, Williams, Schelini, Bazon e Piñon (2013).

d) Inventário de Depressão de Beck (BDI, Beck Depression Inventory) (Beck, Rush, Shaw, \& Emery, 1979): inventário contendo 21 questões, frequentemente usado em pesquisas e intervenções clínicas para se avaliar os níveis de depressão. Foi validado para o Brasil por Cunha (2001);

e) Registro Diário de Bem-Estar e de Senso de Competência Parental: essa ficha contém um continuum de 0 a 10, sendo 0 "fiquei extremamente triste" e 10 "fiquei extremamente feliz", como forma de avaliação do bem-estar (Módulo I); na mesma folha, há outro continuum de 0 a 10 , no qual 0 significa "me senti uma péssima mãe" e 10 "me senti uma ótima mãe", como forma de avaliação de sua competência parental (Módulo II) (Williams et al., 2010); f) Avaliação do Programa pelas participantes: foram realizadas avaliações a fim de verificar a satisfação das participantes em relação ao programa de intervenção. Essas perguntas estão contidas na última parte de cada módulo e as mães deveriam opinar acerca dos aspectos positivos e negativos do material e da intervenção, o que mudou em sua vida como mulher e mãe, além de apontar sugestões.

Foi conduzido um delineamento intrassujeitos com as nove participantes do estudo, sendo que para as participantes 1, 2 e 3, utilizou-se o tratamento simultâneo (pré-teste; intervenção 1 e 2 aplicadas simultaneamente; pós-teste; e follow-up); para as participantes 4, 5 e 6, utilizou-se o tratamento múltiplo (pré-teste; intervenção 1; intervenção 2; pós-teste; e follow-up); e, para as participantes 7, 8 e 9, utilizou-se o tratamento múltiplo (pré-teste; intervenção 2; intervenção 1; pós-teste; e follow-up) (Lourenço, Hayashi, \& Almeida, 2009).

\section{Procedimentos}

Foram realizados 20 encontros individuais com cada participante, sendo que as duas primeiras sessões envolveram coleta de informações gerais e aplicação dos instrumentos; 16 sessões de intervenção apoiadas nos manuais (oito sessões cada); uma sessão para aplicação dos instrumentos no momento pós-teste; e uma sessão de follow-up (três meses após a aplicação do pós-teste). A pesquisa foi aprovada pelo Comitê de Ética em Pesquisa em Seres Humanos da Universidade (Certificado de Apresentação para Apreciação Ética $n^{\circ}$ 0167.0.135.000-08) e as participantes somente integraram o programa de intervenção após terem assinado o Termo de Consentimento Livre e Esclarecido.

O passo seguinte envolveu a realização da Entrevista Inicial com mães vítimas de violência doméstica, com duração de aproximadamente 60 minutos, e a aplicação dos instrumentos BDI, CAP e IEP. Com base nas informações coletadas sobre a renda, a escolaridade e a condição de permanecerem ou não com o parceiro, as nove participantes foram distribuídas, da maneira mais equiparada 
possível, em três grupos. Em seguida, os tipos de procedimentos foram sorteados entre os grupos.

A intervenção do Projeto Parceria foi conduzida individualmente com cada mãe em sessões semanais com duração de 50 minutos cada. Em todos os grupos, foram aplicados os dois módulos, porém com diferença na sequência de aplicação. Sendo assim, no Grupo A, foi realizado o Módulo I e, em seguida, o II; no Grupo B, primeiramente o Módulo II e, após este, o Módulo l; e, no Grupo C, foram realizados os dois módulos simultaneamente (i.e., uma sessão do Módulo I seguida da aplicação de uma sessão do Módulo II na semana seguinte, e assim por diante).

O Módulo I (Williams, Maldonado, \& Padovani, 2008) aborda temas referentes à história de vida da participante, crenças sociais que perpetuam a violência, direitos humanos, medidas de proteção e segurança, redes de apoio, combate à depressão e prevenção de suicídio, o impacto da violência no desenvolvimento humano e treino de habilidades sociais. Os procedimentos utilizados envolveram discussões com base em leituras, relaxamento, resolução de problemas sociais e role-playing. O Módulo II (Williams, Maldonado, \& Araújo, 2008) aborda técnicas de manejo comportamental com crianças, visando o ensino de habilidades parentais positivas: o poder da atenção contingente, técnicas de reforçamento diferencial, consequências justas e eficazes ao comportamento inadequado, estabelecimento de limites e regras, uso do time-out, sistema de fichas etc. Esse módulo foi apresentado com base em informações e discussões, role-playing, leituras, lições de casa e registro de comportamento.

Desde o primeiro até o último encontro, sendo este de pós-teste, foi utilizado um instrumento de medida contínua, o Registro Diário de Bem-Estar e Senso de Competência Parental. A participante levava para casa a folha de registro, atribuindo a si mesma uma nota diária para cada categoria. A folha de registro era entregue à terapeuta semanalmente, em cada sessão, sendo que as duas primeiras semanas de registro, anteriores à intervenção, foram consideradas linha de base.

Para maximizar a adesão, foram utilizadas 714 estratégias bem-sucedidas apontadas por Rios e
Williams (2008): fornecimento de passes para transporte; encontros realizados em local e horário convenientes para as participantes; chamadas telefônicas entre as sessões para que, caso não confirmassem a presença, fosse possível remarcar a sessão para a mesma semana, de preferência; e promoção de incentivos (brindes) de acordo com a frequência nas sessões.

Ao final de ambos os módulos, foram realizadas as avaliações do programa com as participantes e, na última sessão, foram aplicados os mesmos instrumentos do pré-teste (BDI, CAP e IEP). Concluída essa fase, foi realizado o procedimento de follow-up após três meses do término da intervenção, sendo que as mães não participaram de processos terapêuticos no período de espera. No follow-up, foram aplicados os mesmos instrumentos do pós-teste, com exceção do Registro Diário, pois dependeria do acompanhamento semanal do terapeuta. Com base nos resultados do follow-up, as participantes que ainda requeriam cuidados especiais quanto à saúde mental e/ou outros riscos associados à violência foram encaminhadas para acompanhamento psicoterapêutico por estagiários do curso de Psicologia da Universidade.

\section{Resultados e Discussão}

Primeiramente, cabe destacar que todas as participantes aderiram à intervenção, o que é encorajador considerando as variáveis de risco e dificuldade de engajamento à intervenção associadas às participantes (Rios \& Williams, 2008). A adesão pode ser explicada devido aos efeitos benéficos do Projeto Parceria em si, bem como das estratégias utilizadas para maximizá-la. Os resultados são apresentados e discutidos na seguinte sequência: caracterização das participantes; avaliações episódicas (instrumentos BDI, CAP e IEP); avaliações contínuas (registros diários); e avaliação das participantes sobre o Projeto Parceria.

\section{Caracterização das participantes}

Com base nos dados da Entrevista Inicial com mães vítimas de violência doméstica, foi realizada 
uma análise das respostas por meio de Roteiro de Sistematização dos Dados. Essas informações foram agrupadas de acordo com os seguintes tópicos: informações gerais; contexto da violência (permanência com o parceiro, razões que levam/levavam a mãe a permanecer com o agressor, rede de apoio/ opinião acerca da violência); percepção sobre si mesma; condições de saúde da mãe; a infância e a família da participante; e o relacionamento com seus filhos.

De acordo com a Tabela 1, a idade das participantes variou entre 21 e 51 anos, com média de 39 anos, e o número de filhos variou entre 1 e 6, com média de 3,1 filhos. Três mulheres eram casadas, outras três solteiras, duas separadas e uma era viúva. A escolaridade das participantes variou entre 5 e 15 anos de estudo, com média de 10,1 anos, e a renda variou entre 0 e 1 salário-mínimo (salário-mínimo vigente na época da pesquisa: $R \$ 510,00$ ). Sobre o contexto de violência, observou-se que seis das nove participantes permaneciam no relacionamento com o agressor. Elas relatavam ter medo dos parceiros e não se consideravam no controle da agressão, mas nutriam sentimentos de afeto pelos mesmos. Acreditavam que era necessário se separar, mas não se sentiam capazes de se manter financeiramente caso o fizessem.
A maioria das participantes relatou sofrer violência do parceiro desde os primeiros anos do relacionamento, envolvendo tanto a modalidade física quanto a psicológica. Adicionalmente, quatro mulheres relataram ter sofrido violência sexual. A gravidade da violência foi experienciada em seu maior nível (6) por uma das participantes; cinco delas foram avaliadas no nível 4; uma no 3 e duas no 2. A frequência das agressões era constante para quatro delas (diária/semanal) e eventual para cinco (esporádica/mensal). Em geral, elas não possuíam informações sobre como se proteger ou tampouco possuíam apoio de amigos. Acreditavam que não mereciam a violência sofrida e, no entanto, se culpavam.

Essas informações gerais e contexto de violência das participantes apresentam alguns dos fatores de risco vivenciados por elas: baixa renda; baixa escolaridade; permanência com o parceiro agressor; e pouco suporte social para auxiliá-las a enfrentar a situação de violência em que viviam, como, por exemplo, meios de proteção, informações sobre direitos humanos, a importância da denúncia, entre outras. Esses dados corroboram o que é observado na literatura das mulheres vítimas de violência pelo parceiro íntimo (Saffioti, 1997; Soares, 1998; Walker, 1979). Além disso, os dados constatam a manutenção do ciclo da violência pelo par-

Tabela 1

Informações gerais e contexto de violência das participantes

\begin{tabular}{|c|c|c|c|c|c|c|c|c|c|}
\hline Categorias & P1 & $\mathrm{P} 2$ & P3 & P4 & P5 & P6 & P7 & P8 & P9 \\
\hline Idade & 43 & 48 & 34 & 35 & 34 & 51 & 21 & 44 & 41 \\
\hline$N^{\circ}$ filhos & 4 & 2 & 3 & 2 & 3 & 4 & 1 & 6 & 3 \\
\hline Escolaridade (anos) & 11 & 15 & 5 & 11 & 8 & 11 & 11 & 8 & 11 \\
\hline Renda (salário-mínimo) ${ }^{1}$ & 0 & 1 & 1 & 1 & 1 & 0 & 1 & 0 & 1 \\
\hline Permanência c/ parceiro & Não & Sim & Sim & Não & Sim & Sim & Sim & Não & Sim \\
\hline Anos de relacionamento & 12 & 7 & 11 & 8 & 20 & 36 & 5 & 25 & 22 \\
\hline Primeiro episódio (anos relac.) & 10 & 6 & 8 & 7 & 17 & 36 & 3 & 20 & 18 \\
\hline Último episódio & $3 a$ & $6 m$ & $1 a$ & $2 a$ & $3 m$ & 3a & $2 s$ & $6 \mathrm{~m}$ & $4 m$ \\
\hline Tipos de violência & $F, P, S$ & $F, P$ & $F, P$ & $F, P, S$ & $F, P$ & $F, P$ & $F, P, S$ & $F, P, S$ & $F, P$ \\
\hline Grau de violência $(1-6)^{*}$ & 4 & 3 & 2 & 4 & 4 & 4 & 4 & 6 & 2 \\
\hline Frequência da violência & Semanal & Esporádica & Esporádica & Semanal & Esporádica & Mensal & Esporádica & Diária & Semana \\
\hline
\end{tabular}

Nota: 'Salário-mínimo vigente na época da pesquisa: R\$510,00. *Grau de violência definido com base no estudo de McFarlane, Parker, Soeken e Bullock (1992), a saber: 1) Ameaças de agressão, inclusive ameaças com armas; 2) Tapa, empurrão, sem machucar, deixar ferimento ou sem deixar dor duradoura; 3) Soco, chute, machucado/hematoma, cortes e/ou ferimentos com dor duradoura; 4) Espancamento, contusões severas, queimaduras, fraturas de ossos; 5) Ferimentos na cabeça, ferimentos internos ou ferimentos permanentes; 6) Uso de armas, ferimento por armas. a: anos; m: meses; s: semanas; relac.: relacionamento; F: Violência física, P: Violência psicológica e S: Violência sexual. 
ceiro íntimo com a mescla de sentimentos amorosos e de culpa, medo, desejo de separar-se e falta de condições de se manter economicamente sem o parceiro, como exposto por Walker (1979).

Em relação às respostas referentes à percepção das participantes sobre si mesmas, a maioria descreveu pontos negativos relacionados ao humor (e.g., "briguenta", "nervosa"). Tal descrição pode influenciar a maneira de educar os filhos, tendo relação com as dificuldades parentais, assim como observado nos estudos de O'Leary et al. (2000) e Levendosky et al. (2006).

A condição de saúde relatada pelas participantes incluiu queixas de depressão, ansiedade e possíveis sintomas associados, como insônia, gastrite, falta de apetite, problemas de coluna e ideação suicida em oito delas. Esses problemas de saúde enfrentados pela mulher vítima de violência pelo parceiro íntimo também são descritos na metaanálise de Golding (1999).

Seis das nove participantes avaliaram sua infância como "ruim", relatando histórico de violência física e psicológica. A correção dos seus comportamentos inadequados na infância era feita por meio da punição corporal, em sua maioria de autoria materna. Todas as participantes relataram ter dificuldade em manejar o comportamento dos filhos e usaram como justificativa o descontrole emocional ("sou explosiva/estressada/estourada/agressival nervosa") e/ou estilos parentais inadequados ("sou perfeccionista/frouxal, não tenho tempo para cuidar dos meus filhos").

Dessa maneira, o histórico e modelos parentais das participantes podem ter relação com o deficit no repertório parental de suas genitoras (mães das participantes). Assim, o fenômeno observado pode ser considerado como intergeracional. Além disso, a tendência a transferir práticas educativas inadequadas para futuras gerações também foi apontada por Belsky et al. (2009), Serbin e Karp (2003) e Weber et al. (2006).

\section{Análise das avaliações episódicas}

As escalas e inventários foram analisados se-

716 gundo as especificações dos manuais dos respecti- vos instrumentos, de forma a se ter informações sobre o bem-estar (BDI) e a competência parental (IEP e CAP) das participantes no início e no final do estudo, bem como no follow-up. Os dados foram analisados estatisticamente (teste de Wilcoxon) e não foram encontrados resultados significativos entre os três grupos. Sendo assim, a análise desses instrumentos foi realizada por meio do Método JT, proposto por Jacobson e Truax (1991). Optou-se por utilizar esse método em função de ser uma alternativa adequada para a análise de resultados em amostras reduzidas ou em casos de sujeito único. Tal método possibilita a análise quantitativa da validade interna e externa nesses tipos de intervenção, fornecendo informações sobre a variabilidade da resposta ao tratamento para cada paciente em particular, além de indicar a significância clinica do tratamento, isto é, a importância daquelas mudanças na vida dos participantes (Villa, Aguiar, \& Del Prette, 2012).

O Método JT tem por base dois conceitos: Índice de Mudança Confiável, o qual demonstra se a diferença entre o resultado da pós-intervenção e o da pré-intervenção pode ser atribuída ao procedimento realizado e não a um erro de medida; e Significância Clínica, que consiste no nível de funcionamento do participante após a intervenção (Villa et al., 2012). Para demonstrar esses conceitos, são propostas fórmulas, considerando: (a) as diferenças pré e pós-intervenção, para verificar se houve ou não uma mudança confiável; e (b) pontos de corte e intervalo de confiança para a investigação da significância clínica, isto é, se a mudança clínica está generalizada no cotidiano do cliente, possibilitando ter alta da psicoterapia. Por serem indicadores quantitativos, foram analisados por meio de gráficos e a interpretação foi baseada nas instruções de Villa et al. (2012). As análises realizadas por meio do Método JT foram feitas individualmente e por grupo. Cabe ressaltar que as análises referentes aos dados coletados do IEP estão apresentadas com base nas categorias estipuladas pelo instrumento, previamente mencionadas.

Observa-se, na Tabela 2, que o Grupo C concentrou maior número de resultados relacionados à Significância Clínica e Mudança Positiva Confiável, 
Mudança positiva confiável e significância clínica nos instrumentos BDI, CAP e IEP

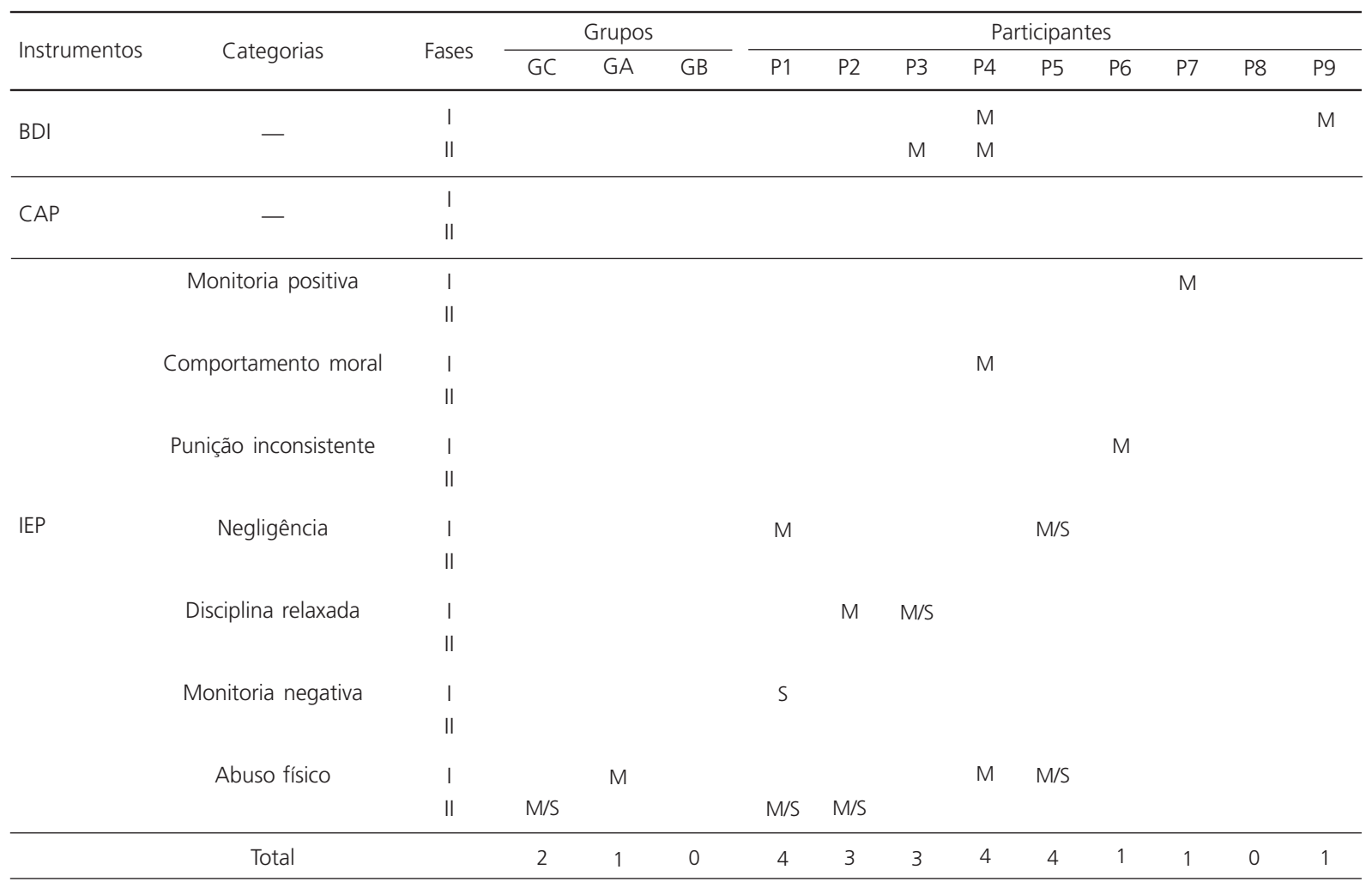

Nota: BDI: Beck Depresion Inventory- Inventário Beck de Depressão; CAP: The Child Abuse Potencial Inventory - Inventário de Potencial de Abuso Infantil; IEP: Inventário de Estilos Parentais; I: Pré/Pós; II: Pós/Follow-up; M: Mudança positiva confiável; S: Significância clínica.

uma vez que as participantes desse grupo (P1, P2 e P3) foram as que mais apresentaram resultados significativos. No Grupo B, não foram verificados resultados significativos. Todas as participantes (com exceção de P8) apresentaram algum escore de mudança positiva confiável ou significância clínica, o mesmo apresentado pelas análises em grupo.

O fato de P8 não ter tido qualquer resultado significativo pode ser explicado pela condição grave vivida pela participante ao ingressar na pesquisa: havia se mudado de cidade em função do parceiro agressor, com o qual vivia há 25 anos, tendo seis filhos. Esse parceiro a violentou sexualmente e ameaçou a família com uma arma. Nessa ocasião, a polícia foi acionada e a participante relatou ter fugido com os filhos para outra cidade. No momento da primeira sessão, quatro meses haviam se passado desde que se mudara para a nova cidade, e a participante ainda estava abalada, com saúde frágil e tentando se adaptar às novas condições com seus filhos. Em função de sua situação crítica, foram realizadas duas sessões de relaxamento e orientações específicas de apoio emocional, mas sem abordar os temas contidos no Módulo I - uma vez que o grupo ao qual pertencia era o que seguia o procedimento Módulo II seguido de Módulo I (Grupo B).

\section{Análise das avaliações contínuas}

O Registro Diário do Bem-Estar da participante e o Registro Diário do Senso de Competência Parental foram coletados semanalmente ao longo do programa, sendo uma medida contínua a qual visava auxiliar na elucidação das possíveis diferenças entre os grupos. Os registros referentes às duas primeiras semanas foram considerados como linha de base (LB), as oito semanas seguintes como a primeira parte da intervenção (T1) e as oito semanas finais como a segunda parte (T2). 
Para testar o efeito de grupo, tempo e a interação entre grupo e tempo, nas variáveis bem-estar e competência parental, utilizou-se o teste denominado Análise de Variância (Anova) não-paramétrica de dados ordinais com medidas repetidas (Brunner \& Langer, 2000). Ora foi utilizada a abordagem com dois fatores (two way) ora com um fator (one way). As comparações entre os períodos da intervenção foram realizadas por meio de testes dois a dois. O nível de significância adotado para os testes foi de $5 \%$.

\section{Bem-estar}

Na Tabela 3, nota-se que as médias aumentaram gradativamente ao longo das semanas em todos os grupos, especialmente no Grupo C. Porém, ao realizar o teste Anova two way, observou-se que não houve diferença significativa entre os grupos $(p=0,7996)$. Houve, então, um esforço no sentido de agrupar os escores dos três grupos e analisá-los nos três momentos do programa (LB, T1 e T2). Dessa maneira, notou-se uma diferença significativa na comparação entre os períodos do programa ( $p=0,0014)$. Os cálculos do teste Anova one way na variável tempo confirmaram a diferença significativa entre os períodos do programa $(p=0,0025)$. Portanto, conforme apresentado na Tabela 3, comparando-se os períodos do programa quanto à variável Bem-estar, foram verificadas diferenças significativas entre a linha de base e a segunda parte da intervenção (LB e T2) e entre a primeira e a segunda parte da intervenção (T1 e T2).

\section{Competência parental}

Assim como observado na variável Bem-Estar, a Tabela 3 nos aponta que os grupos apresentaram médias próximas na variável Competência Parental, com aumento no valor da média ao longo do programa. Aplicando-se o teste Anova two way, observou-se que não houve diferença significativa entre os grupos $(p=0,9600)$, porém houve diferença significativa na comparação entre os períodos do programa $(p=0,0004)$. Ao realizar o teste Anova one way, quanto à variável tempo, foi confirmada a diferença significativa entre os períodos do programa $(p=0,0006)$. Dessa maneira, conforme apresentado na Tabela 3, ao comparar os três períodos do programa quanto à variável Competência Parental, observou-se que houve diferenças significativas em todos os períodos analisados. De acordo com a análise de autorregistro refletindo medidas contínuas de bem-estar e competência Parental pelas mães, pode-se dizer que o Projeto Parceria beneficiou os três grupos indistintamente.

\section{Avaliação do Programa pelas participantes}

Os dados da Avaliação do Programa pelas participantes foram analisados de forma qualitativa por meio de Análise de Conteúdo (Bardin, 1977). As mães relataram terem aproveitado o programa de maneira satisfatória e fizeram vários elogios. A verbalização de P6 ("Eu achei muito importante, me fez muito bem. Tive a impressão que o manual foi feito para $\mathrm{mim}^{\prime \prime}$ ) indica que o conteúdo abordado

Tabela 3

Análises dos registros diários por grupos e períodos

\begin{tabular}{|c|c|c|c|c|c|c|c|}
\hline \multirow{2}{*}{ Variáveis } & \multirow{2}{*}{ Fases } & \multicolumn{3}{|c|}{ Médias das notas } & \multicolumn{3}{|c|}{ Comparações entre períodos } \\
\hline & & Grupo A & Grupo B & Grupo C & LBxT1 & LBxT2 & $\mathrm{T} 1 \mathrm{xT2}$ \\
\hline \multirow[t]{3}{*}{ Bem-Estar } & LB & 6,43 & 5,12 & 5,76 & \multirow{3}{*}{0,1609} & \multirow{3}{*}{$0,0006^{*}$} & \multirow{3}{*}{$0,0015^{*}$} \\
\hline & $\mathrm{T} 1$ & 6,92 & 5,80 & 7,05 & & & \\
\hline & $\mathrm{T} 2$ & 7,62 & 7,40 & 8,18 & & & \\
\hline \multirow[t]{3}{*}{ Competência Parental } & LB & 5,60 & 5,45 & 5,43 & \multirow{3}{*}{$0,0111^{*}$} & \multirow{3}{*}{$0,0000^{*}$} & \multirow{3}{*}{$0,0046^{*}$} \\
\hline & $\mathrm{T} 1$ & 7,28 & 5,73 & 7,24 & & & \\
\hline & $\mathrm{T} 2$ & 7,70 & 7,47 & 7,98 & & & \\
\hline
\end{tabular}

Nota: ${ }^{*}$ Considerando nível de significância $p<0,05$. 
estava em congruência com o perfil da mulher vítima de violência; e a verbalização de P9 ("Foi tão bom que melhorou o ambiente dentro de casa e até com meus amigos") sugere a generalização do tratamento proposto por este módulo ao ambiente da participante. Os aspectos negativos são condizentes com as dificuldades sofridas pelas participantes (como o deficit do repertório, que dificulta colocar em prática algumas situações ensinadas, além da rememoração da condição aversiva sofrida, provocando sentimentos negativos).

\section{Considerações Finais}

O presente estudo atingiu seu objetivo de avaliar os efeitos de três procedimentos diferentes de intervenção cognitivo-comportamental a mulheres com histórico de violência pelo parceiro íntimo em termos das variáveis Bem-Estar e Senso de Competência Parental. Com base no exposto, pode-se concluir que o presente estudo traz uma contribuição relevante acerca dos procedimentos de intervenção com mulheres vítimas de violência pelo parceiro íntimo, com o objetivo de prevenir problemas de comportamento em seus filhos. Além disso, foram observados efeitos favoráveis da intervenção nos três grupos avaliados, especialmente no que recebeu o procedimento simultâneo de módulos (Grupo C).

Adicionalmente, o estudo permitiu confirmar que as participantes - mães com histórico de violência pelo parceiro íntimo -, apresentavam queixas concomitantes em duas áreas principais: (a) dificuldades psicossociais decorrentes da violência conjugal; e (b) dificuldades ao educar seus filhos. Nesse sentido, postula-se que a população desse perfil necessita de apoio especializado em ambas as áreas de forma simultânea, pois, apesar de se ter observado que os três procedimentos contribuíram para uma melhora do desempenho das participantes nas variáveis Bem-estar e Competência parental ao final da intervenção, o grupo submetido ao tratamento simultâneo apresentou uma maior concentração de resultados significativos.

Cabe destacar a utilização de medidas episódicas e contínuas para a coleta de dados deste estu- do. O BDI, IEP e CAP coletados nos momentos pré-teste, pós-teste e follow-up, foram complementados pelas avaliações dos Registros Diários coletados semanalmente. Assim, foi possível verificar se as mudanças no desempenho das participantes, indicadas pelas medidas episódicas, eram acompanhadas ou não pelas medidas contínuas. Além disso, as avaliações das participantes ao final de cada Módulo foram essenciais para se obter um feedback sobre a aceitabilidade do Projeto Parceria.

No entanto, foram observadas algumas limitações do estudo. Uma delas deve-se ao fato de terem sido consideradas as variáveis: renda, escolaridade e a permanência com o parceiro para distribuir as participantes em grupos, mas não foi controlada a gravidade da violência sofrida. Além disso, outras limitações se referem à falta de medidas adicionais as quais poderiam ser importantes para uma análise mais completa, como: dados observacionais da interação mãe-filho; a aplicação de instrumento com os filhos dessas mães, contemplando informações sobre a interação mãe-filho; e, também, aplicação de instrumento com os professores dessas crianças, buscando coletar dados acerca do comportamento em sala de aula. Outra limitação, já mencionada, seria a do próprio Método JT, utilizado para analisar os dados dos instrumentos BDI, CAP e IEP, o qual necessita de maiores pesquisas sobre a comprovação efetiva do Método.

Algumas sugestões podem ser feitas para estudos futuros, como a ampliação do Projeto Parceria com vários profissionais atuando em nesse tipo de intervenção e, com isso, conseguir agregar maior número de participantes. Em relação às medidas de coleta de dados, considerou-se relevante reavaliar algumas questões da Entrevista Inicial a Mulheres Vítimas de Violência Doméstica no pós-teste, especificamente aquelas relacionadas ao bem-estar da participante, seu relacionamento com o parceiro e com seus filhos. Além disso, parece importante acrescentar instrumentos de avaliação pré e pós-teste também com os filhos das participantes, como forma de comparar as respostas das mães, bem como averiguar a melhora ou piora no desempenho das crianças, além de instrumentos com os professores das mesmas. 
Segundo Kazdin (2003), para avaliar a significância clínica de mudanças obtidas em uma intervenção terapêutica, é preciso considerar três indicadores: (a) a diferença entre as avaliações finais e as da pré-intervenção; (b) o impacto social em medidas reconhecidamente relevantes de ajustamento psicológico, como evasão/adesão; e (c) a avaliação subjetiva do cliente ou de seus significantes. A partir das análises realizadas, associadas às interpretações do Método JT, observou-se que o Grupo C concentrou melhores resultados. Em relação ao segundo dado, sobre o impacto social, verificou-se que houve adesão de todas as participantes, sendo possível inferir que a potencialidade do programa, aliada às estratégias utilizadas, foi adequada para manter as participantes até o final da intervenção. Por fim, de acordo com as avaliações de satisfação das participantes sobre o programa, verificou-se que as respostas foram todas positivas e com algumas sugestões importantes a serem consideradas em uma futura aplicação.

Na última década, o tema da violência esteve vinculado à saúde, no que se refere à realidade brasileira (Brasil, 2002), enfatizando a necessidade de envolvimento e preparo dos profissionais e dos serviços de saúde, dada a conjuntura complexa e desafiante do tema. Entretanto, é fundamental que os serviços (postos, centros de saúde, hospitais, resgates) e profissionais reconheçam que devem fazer parte do processo de prevenção, na identificação de condições de risco ou de violência vigente e na atenção às vítimas.

O Projeto Parceria é um programa de intervenção com resultados importantes, destacando sua relevância social e científica. Com base nos resultados indicados neste estudo, concluiu-se que o procedimento adequado a ser utilizado com participantes do perfil estudado deve ser a aplicação simultânea de psicoterapia focada no histórico de violência pelo parceiro íntimo à orientação sobre práticas parentais educativas positivas. Dessa maneira, um programa o qual aborde o procedimento indicado pode ser replicado em outras instituições de apoio às mulheres vítimas de violência pelo parceiro íntimo com chances de sucesso, salientando seu provável

\section{Colaboradores}

Todos os autores contribuíram na concepção e desenho do estudo, análise de dados e redação final.

\section{Referências}

Appel, A. E., \& Holden, G. W. (1998). The co-occurrence of spousal and physical child maltreatment: A review and appraisal. Journal of Family Psychology, 12(4), 578-599.

Bardin, L. (1977). Análise de conteúdo. Portugal: Edições 70.

Beck, A. T., Rush, A. J., Shaw, B. F., \& Emery, G. (1979). Cognitive therapy of depression: A treatment manual. New York: Guilford Press.

Belsky, J., Conger, R., \& Capaldi, D. M. (2009). The intergenerational transmission of parenting: Introduction to the special section. Developmental Psychology, 45(5), 1201-1204.

Brasil. Ministério da Saúde. (2002). Violência intrafamiliar: orientações para a prática em serviço. Brasília: Autor. (Série Cadernos de Atenção Básica, ${ }^{\circ} 8$, e Série A: Normas e Manuais Técnicos, $n^{\circ}$ 131). Recuperado em fevereiro 15, 2013, de http://bvsms.saude.gov.br/bvs/ publicacoes/cd05_19.pdf

Brunner, E., \& Langer, F. (2000). Nonparametric analysis of ordered categorical data in designs with longitudinal observations and small sample sizes. Biometrical Journal, 42(6), 663-675.

Casanueva, C., Martin, S. L., \& Runyan, D. (2009). Repeated reports of child maltreatment among intimate partner violence victims: Findings from the national survey of child and adolescent well-being. Child Abuse and Neglect, 33(2), 84-93.

Cunha, J. A. (2001). Manual da versão em português das Escalas Beck. São Paulo: Casa do Psicólogo.

Golding, J. M. (1999). Intimate partnet violence as a risk fator for mental disorders: A meta-analysis. Journal of Family Violence, 14(2), 99-132.

Gomide, P. I. C. (2006). Inventário de Estilos Parentais (IEP). Petrópolis: Vozes.

Jacobson, N. S., \& Truax, P. (1991). Clinical significance: A statistical approach to defining meaningful change in psychotherapy research. Journal of Consulting and Clinical Psychology, 59(1), 12-19.

Jouriles, E. N., McDonald, R., Rosenfield, D., Stephens, N., Corbitt-Shindler, D., \& Miller, P. C. (2009). Reducing conduct problems among children exposed to intimate partner violence: A randomized clinical trial examining effects of project support. Journal of Consulting and Clinical Psychology, 77(4), 705-717. 
Kazdin, A. E. (2003). Research design in clinical psychology (4 ${ }^{\text {th }}$ ed.). Boston: Allyn \& Bacon.

Levendosky, A. A., Leahy, K. L., Bogat, G. A., Davidson, W. S., \& Von Eye, A. (2006). Domestic violence, maternal parenting, maternal mental health, and infant externalizing behavior. Journal of Family Psychology, 20(4), 544-552.

Lourenço, E. A. G., Hayashi, M. C. P. I., \& Almeida, M. A. (2009). Delineamentos intrassujeitos nas dissertações e teses do PPGEES/UFSCAR. Revista Brasileira de Educação Especial, 15(2), 319-336.

McFarlane, J., Parker, B., Soeken, K., \& Bullock, L. (1992). Assessing for abuse during pregnancy. Severity and frequency of injuries and associated entry into prenatal care. Journal of the American Medical Association, 267(23), 3176-3178.

Milner, J. S. (1994). Assessing physical child abuse risk: The child abuse potential inventory. Clinical Psychology Review, 6(14), 547-583.

O'Leary, K. D., Slep, A. M. S., \& O'Leary, S. G. (2000). Cooccurrence of partner and parent aggression: Research and treatment implications. Behavior Therapy, 31(4), 631-648.

Patrian, A. C. A., Rios, K. S. A., \& Williams, L. C. A. (2013). Validade de critério do Inventário de Potencial para Abuso Infantil (CAP). Paidéia, 23(54), 43-51.

Pereira, P. C., D'Affonseca, S. M., \& Williams, L. C. A. (2013). A feasibility pilot intervention program to teach parenting skills to poly-victimized children. Journal of Family Violence, 28(1), 5-15.

Rios, K. S. A., \& Williams, L. C. A. (2008). Intervenção com famílias como estratégia de prevenção de problemas de comportamento em crianças: uma revisão. Psicologia em Estudo, 13(4), 799-806.

Rios, K. S. A., Williams, L. C. A., Schelini, P. W., Bazon, M. R., \& Piñon, E. A. (2013). Inventário de Potencial de Abuso Infantil - CAP: evidências de validade e precisão. Avaliação Psicológica, 12(1), 51-60.

Saffioti, H. L. B. (1997). Violência doméstica ou a lógica do galinheiro. In M. Kuptskas (Ed.), Violência em debate. São Paulo: Moderna.

Serbin, L., \& Karp, J. (2003). Intergenerational studies of parenting and the transfer of risk from parent to child. American Psychological Society, 12(4), 138-142.

Soares, B. M. (1998). Mulheres invisíveis. Rio de Janeiro: Civilização Brasileira.

Sullivan, C. M. (2006). Interventions to address intimate partner violence: The current state of the field. In J. R. Lutzker (Ed.), Preventing violence: Research and evidence-based intervention strategies (pp. 195-212). Washington: American Psychological Association.

Villa, M. B., Aguiar, A. A. R., \& Del Prette, Z. A. P. (2012). Intervenções baseadas em evidências: aplicações do método JT. São Carlos: EdUFSCar.

Walker, L. (1979). The battered woman. New York: Harper and Row.

Weber, L. N. D., Selig, G. A., Bernardi, M. G., \& Salvador, A. P. V. (2006). Continuidade dos estilos parentais através de gerações: transmissão intergeracional dos estilos parentais. Paidéia, 16(35), 407-441.

Williams, L. C. A. (2010). Entrevista inicial com mulheres vítimas de violência doméstica. In L. C. A. Williams, J. M. D. Maia, \& K. S. A. Rios (Eds.), Aspectos psicológicos da violência: pesquisa e intervenção cognitivo-comportamental (pp.549-558). Santo André: ESETec.

Williams, L. C. A, Araújo, E. A. C., Rios, K. S. A., D'Affonseca, S. M., Maldonado, D. P. A., Patrian, A. C. A., \& Miranda, T. D. (2010). Projeto Parceria: o ensino de habilidades parentais a mães com histórico de violência conjugal. In L. C. A Williams, J. M. D. Maia, \& K. S. A. Rios (Eds.), Aspectos psicológicos da violência: pesquisa e intervenção cognitivo-comportamental (pp.428-457). Santo André: ESETec.

Williams, L. C. A., Maldonado, D. P. A., \& Araújo, E. A. C. (2008). Educação positiva dos seus filhos: Projeto Parceria - módulo 2. São Carlos: UFSCar. Recuperado em maio 12, 2014, de http://www.laprev.ufscar.br/ documentos/arquivos/apostilas-e-manuais/parceria2.pdf

Williams, L. C. A., Maldonado, D. P. A., \& Padovani, R. C. (2008). Uma vida livre de violência: Projeto Parceria módulo 1. São Carlos: UFSCar. Recuperado em maio 12, 2014, de http://www.laprev.ufscar.br/documentos/ arquivos/apostilas-e-manuais/parceria-1.pdf

Williams, L. C. A., Santini, P. M., \& D'Affonseca, S. M. (2012). A mothering skills program for women with a history of domestic violence: An analysis of mother-child interaction. In H. Dubowitz (Ed.), World perspectives on child abuse (10 ${ }^{\text {th }}$ ed., pp.134-136). Istanbul: International Society for the Prevention of Child Abuse and Neglect.

Williams, L. C. A., Santini, P. M., \& D’Affonseca, S. M. (2014). The Parceria Project: A Brazilian parenting program to mothers with a history of intimate partner violence. International Journal of Applied Psychology, 4(3), 101-107.

Recebido: fevereiro 25, 2014

Versão final: maio 21, 2014

Aprovado: junho 9, 2014 
